\title{
Lower bounds for graph bootstrap percolation via properties of polynomials
}

\author{
Lianna Hambardzumyan * Hamed Hatami ${ }^{\dagger} \quad$ Yingjie Qian ${ }^{\ddagger}$
}

\begin{abstract}
We introduce a simple method for proving lower bounds for the size of the smallest percolating set in a certain graph bootstrap process. We apply this method to obtain recursive formulas for the sizes of the smallest percolating sets in multidimensional tori and multidimensional grids (in particular hypercubes). The former answers a question of Morrison and Noel [MN18, and the latter provides an alternative and simpler proof for one of their main results.
\end{abstract}

Keywords: Bootstrap, Percolation, graph bootstrap.

\section{Introduction}

Graph bootstrap processes arise naturally in statistical mechanics, probability theory, combinatorics, and social sciences, and thus have been extensively studied in the past four decades or so. In these processes, one starts with an initial set of infected vertices (or sites) or edges (or bonds) in a graph, and at every step, the infection spreads to a new vertex or edge according to some local rule. The goal is often to understand the properties of the percolating sets, i.e. the initial sets of infected vertices or edges for which the infection eventually spreads to all the vertices or edges.

The most commonly studied notion of bootstrap percolation is the r-neighbour bootstrap percolation, introduced in [CLR79] in the context of disordered magnetic systems in statistical mechanics. In this process, one starts with an initial set of infected vertices, and at every step, the infection spreads to the vertices that have at least $r$ infected neighbours. While the main focus of the research that is motivated by problems in statistical physics has been on determining the critical threshold at which a random initial infected set percolates, fundamental extremal problems such as determining the size of the smallest percolating sets have been investigated extensively as well. Indeed this problem is often closely related to the problem of determining the critical percolation threshold [BBMR12, BBM10, GHM12, BB06]. We will denote the size of the smallest percolating set in a graph $G$ in the $r$-neighbour bootstrap percolation process by $m(G, r)$.

In this paper, we are interested in a closely related bootstrap process, which we refer to as the $r$-bond bootstrap percolation. In this process, we start with a set of infected edges, and at every step, the infection spreads to a new edge if at least one of its endpoints is incident to at least $r$ infected edges. In other words, once a vertex is incident to $r$ infected edges, then the infection spreads to all of the edges that are incident to that vertex. We denote the size of the smallest percolating set for this process by $m_{e}(G, r)$. This natural process seems to have been introduced first in [LZ84 for the two dimensional grid to model how a wetting fluid fills the ducts in the network of a porous media.

The $r$-bond bootstrap percolation is an instance of the graph bootstrap process defined in 1968 by Bollobás [Bol68. Given graphs $G$ and $H$, and an initial set of infected edges, in the $H$-bootstrap process, at each time step, we infect an edge $e$ if it completes a new infected copy of $H$ in $G$. Note that taking $H$

*School of Computer Science, McGill University. lianna.hambardzumyan@mail.mcgill.ca

${ }^{\dagger}$ School of Computer Science, McGill University. hatami@cs.mcgill.ca. Supported by an NSERC grant.

${ }^{\ddagger}$ Department of Mathematics and Statistics, McGill University. yingjie.qian@mail.mcgill.ca 
to be the star with $r+1$ leaves (denoted by $S_{r+1}$ ), results in the above-mentioned process. The size of the smallest percolating set of edges in $G$ in the $H$-percolation process is called the weak saturation number of $H$ in $G$ and is denoted by wsat $(G, H)$. Hence in our notation $m_{e}(G, r)=\operatorname{wsat}\left(G, S_{r+1}\right)$.

Note that one can turn a percolating set of vertices for the $r$-neighbour bootstrap process to a percolating set of edges for the $r$-bond bootstrap process by infecting $r$ arbitrarily chosen edges incident to every initially infected vertex (if the degree of the vertex is less than $r$, then we just infect all the edges incident to it). Similarly, given a percolating set of edges for the $r$-bond bootstrap process, to obtain a percolating set for the $r$-neighbour bootstrap process, one can pick one endpoint of every infected edge; these vertices together with all the vertices of degree less than $r$ form a percolating set of vertices for the $r$-neighbour bootstrap process. These observations show

$$
\frac{m_{e}(G, r)}{r} \leq m(G, r) \leq m_{e}(G, r)+\left|\left\{v: \operatorname{deg}_{G}(v)<r\right\}\right| .
$$

Recently Morrison and Noel [MN18] used (11) to determine the asymptotics of $m\left(Q_{d}, r\right)$, where $Q_{d}$ denotes the $d$-dimensional hypercube. Indeed, they proved the exact formula

$$
m_{e}\left(Q_{d}, r\right)=\sum_{j=1}^{r}\left(\begin{array}{c}
d-j-1 \\
r-j
\end{array}\right) j 2^{j-1}
$$

and combined it with (11) to show that $m\left(Q_{d}, r\right)=\frac{d^{r-1}}{r !}+\Theta_{d \rightarrow \infty}\left(d^{r-2}\right)$, settling a conjecture of [BB06]. Prior to [MN18, the best known lower bounds for $m\left(Q_{d}, r\right)$ were only linear in $d$.

The purpose of this article is to introduce a simple method based on the fact that a non-zero polynomial of degree $r$ has at most $r$ roots for proving lower bounds for $m_{e}(G, r)$. We will use this method to settle a problem of Morrison and Noel MN18, by determining $m_{e}(G, r)$ for the multidimensional tori (Theorem 8). Moreover, we provide an alternative and simpler proof for the case of the hypercube, and more generally, the multidimensional grid (Theorem 9), which were originally established in [MN18.

Applying this property of polynomials to percolation seems very natural, and indeed as an anonymous referee pointed out to us, Balister et al. [BBLN18] have used it recently to determine the size of the minimal percolating set of $d$-dimensional integer grid in a slightly different context of the line percolation model.

\subsection{Notation}

For a positive integer $n$, we denote $[n]=\{1, \ldots, n\}$. For a graph $G=(V, E)$, and an edge colouring $c: E \rightarrow \mathbb{R}$, to simplify the notation we often denote the colour of an edge $e$ by $c_{e}$. An edge colouring is called proper if it assigns different colours to incident edges. For a logical statement $P$, we define $1_{[P]}$ to be 1 if $P$ is true, and 0 if $P$ is false.

The Cartesian product of two graphs $G$ and $H$, denoted by $G \square H$, is the graph with vertex set $V(G) \times$ $V(H)$, in which two vertices $\left(u_{1}, v_{1}\right)$ and $\left(u_{2}, v_{2}\right)$ are adjacent if and only if either $u_{1}=u_{2}$ and $v_{1} v_{2} \in E(H)$, or $v_{1}=v_{2}$ and $u_{1} u_{2} \in E(G)$. In other words, for every vertex $v \in V(H)$, we have a copy $G_{v}$ of $G$, induced on vertices $\{(u, v): u \in V(G)\}$, and for every edge $v_{1} v_{2} \in E(H)$, there is a matching between $G_{v_{1}}$ and $G_{v_{2}}$ that connects each vertex of $G_{v_{1}}$ to its corresponding vertex in $G_{v_{2}}$.

\section{Polynomials and Bootstrap Percolation}

We start with the following key definition.

Definition 1. Let $r \geq 0$ be an integer, $G=(V, E)$ be a graph, and let $c: E \rightarrow \mathbb{R}$ be a proper edge colouring of $G$. Let $W_{G, c}^{r}$ be the vector space of all vectors $\left(p_{v} \in \mathbb{R}[x]: v \in V\right)$, where $p_{v}$ 's are univariate polynomials such that

1. $\operatorname{deg}\left(p_{v}\right) \leq \min \{r, \operatorname{deg}(v)\}-1$ (here we use the convention that the degree of the zero polynomial is -1 ); 
2. $p_{u}\left(c_{u v}\right)=p_{v}\left(c_{u v}\right)$ for every edge $u v \in E$.

Note that $W_{G, c}^{r}$ is indeed a vector space as a set of polynomials satisfying the above conditions is closed under addition and multiplication by scalars.

The following theorem summarizes the main idea of this article.

Theorem 2. Let $c: E \rightarrow \mathbb{R}$ be a proper edge colouring of a graph $G=(V, E)$, and $r \geq 0$ be an integer. We have

$$
m_{e}(G, r) \geq \operatorname{dim}\left(W_{G, c}^{r}\right) .
$$

Proof. Let $F \subseteq E$ be a percolating set for the $r$-bond bootstrap process in $G$. We claim that if a vector $\left(p_{v}\right)_{v \in V} \in W_{G, c}^{r}$ satisfies $p_{u}\left(c_{u v}\right)=p_{v}\left(c_{u v}\right)=0$ for all $u v \in F$, then $p_{v} \equiv 0$ for all $v \in V$. To prove this claim, observe that throughout the process, the condition $p_{u}\left(c_{u v}\right)=p_{v}\left(c_{u v}\right)=0$ will be forced for the newly infected edges $u v$. Note that if a vertex $u$ is incident to at least $\alpha_{u}:=\min \{r, \operatorname{deg}(u)\}$ infected edges, then we know that $p_{u}$ has at least $\alpha_{u}$ distinct roots, as it has to evaluate to 0 on the colours of its neighbouring infected edges. However, since the degree of $p_{u}$ is at most $\alpha_{u}-1$, this implies $p_{u} \equiv 0$, and thus $p_{u}$ evaluates to 0 on all the edges incident to $u$. This corresponds to the spreading of infection to all the edges incident to $u$. Since $F$ percolates, eventually all the polynomials $p_{u}$ in the vector $\left(p_{u}\right)_{u \in V}$ will be forced to be equivalent to 0 .

Now let us show that theorem follows from the above claim. Recall that $\alpha_{v}=\min \{r, \operatorname{deg}(v)\}$ for $v \in V$, and define the vector space $X=\left\{\left(p_{u}\right)_{u \in V} \mid \operatorname{deg}\left(p_{u}\right) \leq \alpha_{u}-1 \forall u \in V\right\}$, and its subspace $Y=\left\{\left(p_{u}\right)_{u \in V} \in\right.$ $\left.X \mid p_{u}\left(c_{u v}\right)=0 \forall u v \in F\right\}$. Note that in the definition of $Y$, we force the condition $p_{u}\left(c_{u v}\right)=0$ only for one endpoint of the edge, which can be chosen arbitrarily. Clearly, $W_{G, c}^{r} \subset X$ and $Y \subset X$, and co-dim( $(Y) \leq|F|$ in $X$. The claim proven in the previous paragraph shows that $W_{G, c}^{r} \cap Y=\{\overrightarrow{0}\}$. Hence

$$
\operatorname{dim}\left(W_{G, c}^{r}\right)+\operatorname{dim}(Y) \leq \operatorname{dim}(X)
$$

which yields the desired bound $\operatorname{dim}\left(W_{G, c}^{r}\right) \leq|F|$.

To warm up let us consider a simple example. Let $G=(V, E)$ be a graph with maximum degree $r$. Obviously, $m_{e}(G, r)=|E|$, as in such a graph, the initial infection cannot spread to any new edges. The following proposition shows that the lower bound provided by Theorem 2 is sharp for such graphs.

Proposition 3. Let $r$ be a non-negative integer, and let $c: E \rightarrow \mathbb{R}$ be a proper edge colouring of a graph $G=(V, E)$ with maximum degree $r$. We have $m_{e}(G, r)=\operatorname{dim}\left(W_{G, c}^{r}\right)=|E|$.

Proof. Consider an edge $e_{0}=u_{0} v_{0} \in E$. Let the vector $\mathbf{p}_{e_{0}}=\left(p_{u}\right)_{u \in V}$ for the edge $e_{0}$ be defined as follows. Let $p_{u_{0}}$ be a polynomial of $\operatorname{degree} \operatorname{deg}\left(u_{0}\right)-1$ that is equal to 1 on $c_{u_{0} v_{0}}$, and is equal to 0 on $c_{u_{0} v}$ for every $u_{0} v \in E \backslash\left\{u_{0} v_{0}\right\}$. Such a polynomial exists since $p_{u_{0}}$ has $\operatorname{deg}\left(u_{0}\right)-1$ roots, and this does not exceed its degree. Similarly, choose $p_{v_{0}}$ to be a polynomial of degree $\operatorname{deg}\left(v_{0}\right)-1$ that is equal to 1 on $c_{u_{0} v_{0}}$ and is equal to 0 on $c_{u v_{0}}$ for every $u v_{0} \in E \backslash\left\{u_{0} v_{0}\right\}$. Set $p_{u} \equiv 0$ for all $u \notin\left\{u_{0}, v_{0}\right\}$. Člearly, the vector $\mathbf{p}_{e_{0}}$, constructed in this way, belongs to $W_{G, c}^{r}$. Next note that the vectors $\left\{\mathbf{p}_{e_{0}}: e_{0} \in E\right\}$ are linearly independent, and thus $\operatorname{dim}\left(W_{G, c}^{r}\right) \geq|E|$. Combining this with Theorem 2 and the trivial inequality $m_{e}(G, r) \leq|E|$, we deduce the statement of the proposition.

\section{Tori and Grids}

In this section, we apply Theorem 2 to obtain recursive formulas for $m_{e}(G, r)$ for tori and grids in arbitrary dimensions. In fact, our results are more general as they apply to the Cartesian products of arbitrary graphs with cycles and paths. The cases of the tori and grids will follow easily from those by simple inductions. First we prove an upper bound on $m_{e}\left(G \square C_{k}, r\right)$ by constructing a percolating set of appropriate size. Here we assume $r>1$, since, trivially, $m_{e}(G, 0)=0$ and $m_{e}(G, 1)=1$ for every connected graph $G$. 
Proposition 4. Let $r>1, k \geq 3$ be integers, and $G=(V, E)$ be a graph. We have

$$
m_{e}\left(G \square C_{k}, r\right) \leq m_{e}(G, r)+(k-2) m_{e}(G, r-1)+m_{e}(G, r-2)+d_{r-1}+k\left(d_{0}+\ldots+d_{r-2}\right),
$$

where $d_{t}$ denotes the number of vertices with degree exactly $t$ in $G$.

Proof. For every vertex $v \in G$, denote its corresponding vertices in $G \square C_{k}$ by $v_{1}, v_{2}, \ldots, v_{k}$. Let $G_{1}, \ldots, G_{k}$ denote the $k$ copies of $G$ in $G \square C_{k}$ corresponding to the $k$ vertices of $C_{k}$. First let us consider the case where every vertex of $G$ is of degree at least $r$. Construct a percolating set $F$ for $G \square C_{k}$ in the following manner. Pick an optimal $r$-percolating set $F_{r}\left(G_{1}\right)$ for $G_{1}$, optimal $(r-1)$-percolating sets $F_{r-1}\left(G_{l}\right)$ for $G_{l}$, $l \in\{2, \ldots, k-1\}$, and an optimal $(r-2)$-percolating set $F_{r-2}\left(G_{k}\right)$ for $G_{k}$.

Since $F_{r}\left(G_{1}\right) \subseteq F$, after running the $r$-bond bootstrap process on $G_{1}$, all the edges in $G_{1}$ will be infected, and then due to the degree condition, the infection will pass to all the edges between $G_{1}$ and $G_{2}$, and $G_{1}$ and $G_{k}$. Now every vertex in $G_{2}$ has an infected edge coming from $G_{1}$. This together with the edges in $F_{r-1}\left(G_{2}\right) \subseteq F$ infects all the edges in $G_{2}$, and consequently all the edges between $G_{2}$ and $G_{3}$ will be infected. Continuing in this manner, all the edges will be infected except possibly the edges inside $G_{k}$. However, at this point, every vertex in $G_{k}$ has two external infected edges incident to it, one from $G_{1}$ and one from $G_{k-1}$. Thus the edges in $F_{r-2}\left(G_{k}\right) \subseteq F$ will eventually infect all the edges in $G_{k}$.

It remains to deal with the vertices of degrees less than $r$. If $\operatorname{deg}_{G}(v)=r-1$, then we only need to add the edge $v_{1} v_{k}$ to the above set. This will guarantee that once $G_{1}$ is fully infected, $v_{1} v_{2}$ will become infected, and the process proceeds as it is described above. Finally, for the vertices with degree $\operatorname{deg}_{G}(v)<r-1$, one can (and must) simply include all the edges $v_{i} v_{i+1}$ for $i=1, \ldots, k$ (let $v_{k+1}=v_{1}$ ).

Now we turn to proving a lower bound for $m_{e}\left(G \square C_{k}, r\right)$. By Theorem 2, it suffices to prove a lower bound for $\operatorname{dim}\left(W_{G \square C_{k}, c^{\prime}}^{r}\right)$ where $c^{\prime}$ is a proper edge colouring of $G \square C_{k}$. This is achieved in Theorem 5 below, which complements Proposition 4

Theorem 5. Let $r>1, k \geq 3$ be integers, and $c: E \rightarrow \mathbb{R}$ be a proper edge colouring of a graph $G=(V, E)$. There exists a proper edge colouring $c^{\prime}$ of $G \square C_{k}$ for which

$$
\operatorname{dim}\left(W_{G \square C_{k}, c^{\prime}}^{r}\right) \geq \operatorname{dim}\left(W_{G, c}^{r}\right)+(k-2) \operatorname{dim}\left(W_{G, c}^{r-1}\right)+\operatorname{dim}\left(W_{G, c}^{r-2}\right)+d_{r-1}+k\left(d_{0}+\ldots+d_{r-2}\right),
$$

where $d_{t}$ denotes the number of vertices with degree exactly $t$ in $G$.

Proof. For every vertex $v \in G$, denote its corresponding vertices in $G \square C_{k}$ by $v_{1}, v_{2}, \ldots, v_{k}$. Let $G_{1}, \ldots, G_{k}$ denote the $k$ copies of $G$ in $G \square C_{k}$ corresponding to the $k$ vertices of $C_{k}$. Let $\alpha_{1}, \ldots, \alpha_{k}$ be distinct real numbers that do not belong to $c(E)$. Let $c^{\prime}$ be the proper colouring of $G \square C_{k}$ that is consistent with $c$ on $G_{1}, \ldots, G_{k}$ and moreover $c^{\prime}\left(v_{i} v_{i+1}\right)=\alpha_{i}$ for all $i \in[k]$ and $v \in V(G)$ (where $v_{k+1}=v_{1}$ ). To prove the theorem, we are going to find $\operatorname{dim}\left(W_{G, c}^{r}\right)+(k-2) \operatorname{dim}\left(W_{G, c}^{r-1}\right)+\operatorname{dim}\left(W_{G, c}^{r-2}\right)+d_{r-1}+k\left(d_{0}+\ldots+d_{r-2}\right)$ linearly independent vectors in $W_{G \square C_{k}, c^{\prime}}^{r}$.

Consider a linear basis $B^{(r)}$ for $W_{G, c}^{r}$. Pick a vector $\mathbf{q} \in B^{(r)}$ and let $\mathbf{q}=\left(q_{v}\right)_{v \in V(G)}$. Define the vector $\mathbf{p}_{\mathbf{q}}^{(1)}=\left(p_{u}\right)_{u \in V\left(G \square C_{k}\right)}$ as $p_{v_{i}}=q_{v}$ for $i \in[k]$ and $v \in V(G)$. (See Figure 1). Trivially, the two conditions in Definition 1 are satisfied, and thus the vector $\mathbf{p}_{\mathbf{q}}^{(1)}$ belongs to $W_{G \square C_{k}, c^{\prime}}^{r}$. Set $B_{1}=\left\{\mathbf{p}_{\mathbf{q}}^{(1)}: \mathbf{q} \in B^{(r)}\right\}$. Note that the restriction of $\mathbf{p}_{\mathbf{q}}^{(1)}$ to $G_{1}$ equals $\mathbf{q}$, and thus the vectors in $B_{1}$ are linearly independent.

Next, consider a linear basis $B^{(r-1)}$ for $W_{G, c}^{r-1}$, and fix $\ell \in\{2, \ldots, k-1\}$. Let $\mathbf{q} \in B^{(r-1)}$ and $\mathbf{q}=$ $\left(q_{v}\right)_{v \in V(G)}$. Note that $\operatorname{deg}\left(q_{v}\right) \leq \min \left\{r-1, \operatorname{deg}_{G}(v)\right\}-1$ for all $v$. Define the vectors $\mathbf{p}_{\mathbf{q}}^{(\ell)}=\left(p_{u}\right)_{u \in V\left(G \square C_{k}\right)}$ as

$$
p_{v_{\ell}}=\frac{x-\alpha_{\ell-1}}{\alpha_{\ell}-\alpha_{\ell-1}} q_{v}, \quad p_{v_{\ell+1}}=\frac{x-\alpha_{\ell+1}}{\alpha_{\ell}-\alpha_{\ell+1}} q_{v}, \quad \text { for all } v \in V(G),
$$

and $p_{v_{j}} \equiv 0$ for all $j \notin\{\ell, \ell+1\}$ and $v \in V(G)$. (See Figure 2). Note that $\operatorname{deg}\left(p_{v_{\ell}}\right) \leq \operatorname{deg}\left(q_{v}\right)+1=\min \{r-$ $\left.1, \operatorname{deg}_{G}(v)\right\}=\min \left\{r-1, \operatorname{deg}\left(v_{\ell}\right)-2\right\} \leq \min \left\{r, \operatorname{deg}\left(v_{\ell}\right)\right\}-1$, and similarly, $\operatorname{deg}\left(p_{v_{\ell+1}}\right) \leq \min \left\{r, \operatorname{deg}\left(v_{\ell+1}\right)\right\}-1$. 


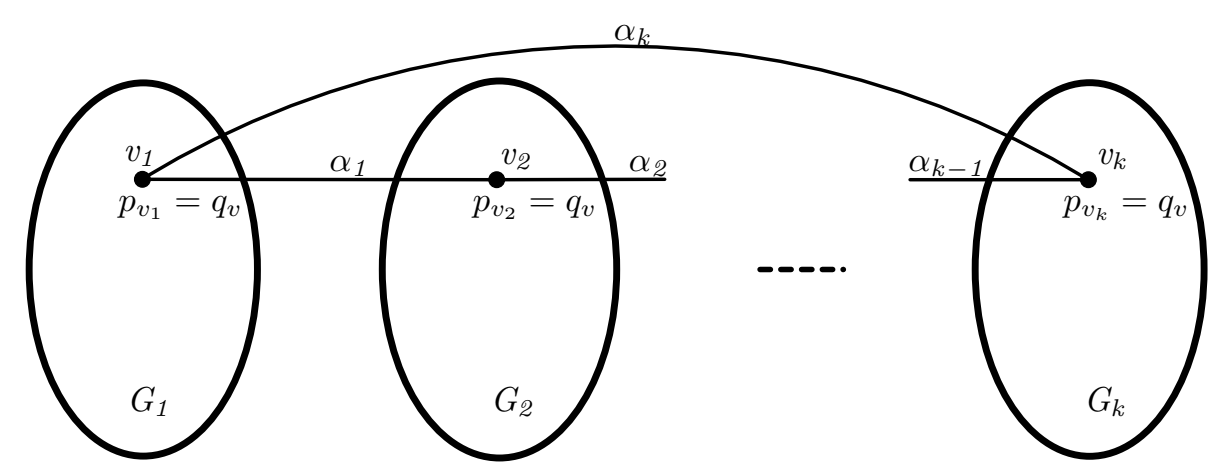

Figure 1: The vector $\left(q_{v}\right)_{v \in V(G)} \in B^{(r)}$ is used to construct $\mathbf{p}_{\mathbf{q}}^{(1)}=\left(p_{u}\right)_{u \in V\left(G \square C_{k}\right)}$ such that $\mathbf{p}_{\mathbf{q}}^{(1)} \in B_{1} \subseteq$ $W_{G \square C_{k}, c^{\prime}}^{r}$

Moreover, $p_{v_{\ell}}\left(\alpha_{\ell}\right)=p_{v_{\ell+1}}\left(\alpha_{\ell}\right)=q_{v}\left(\alpha_{\ell}\right)$, and $p_{v_{\ell}}\left(\alpha_{\ell-1}\right)=p_{v_{\ell+1}}\left(\alpha_{\ell+1}\right)=0$. Hence both conditions in Definition 1 are satisfied, and the vectors $\mathbf{p}_{\mathbf{q}}^{(\ell)}$ belong to $W_{G \square C_{k}, c^{\prime}}^{r}$. Define $B_{\ell}=\left\{\mathbf{p}_{\mathbf{q}}^{(\ell)}: \mathbf{q} \in B^{(r-1)}\right\}$. Note that the restriction of $\mathbf{p}_{\mathbf{q}}^{(\ell)}$ to $G_{\ell}$ is the following vector $\left(\frac{x-\alpha_{\ell-1}}{\alpha_{\ell}-\alpha_{\ell-1}} q_{v}\right)_{v \in V(G)}$, and since $\frac{x-\alpha_{\ell-1}}{\alpha_{\ell}-\alpha_{\ell-1}} \not \equiv 0$, the vectors in $B_{\ell}$ are linearly independent.

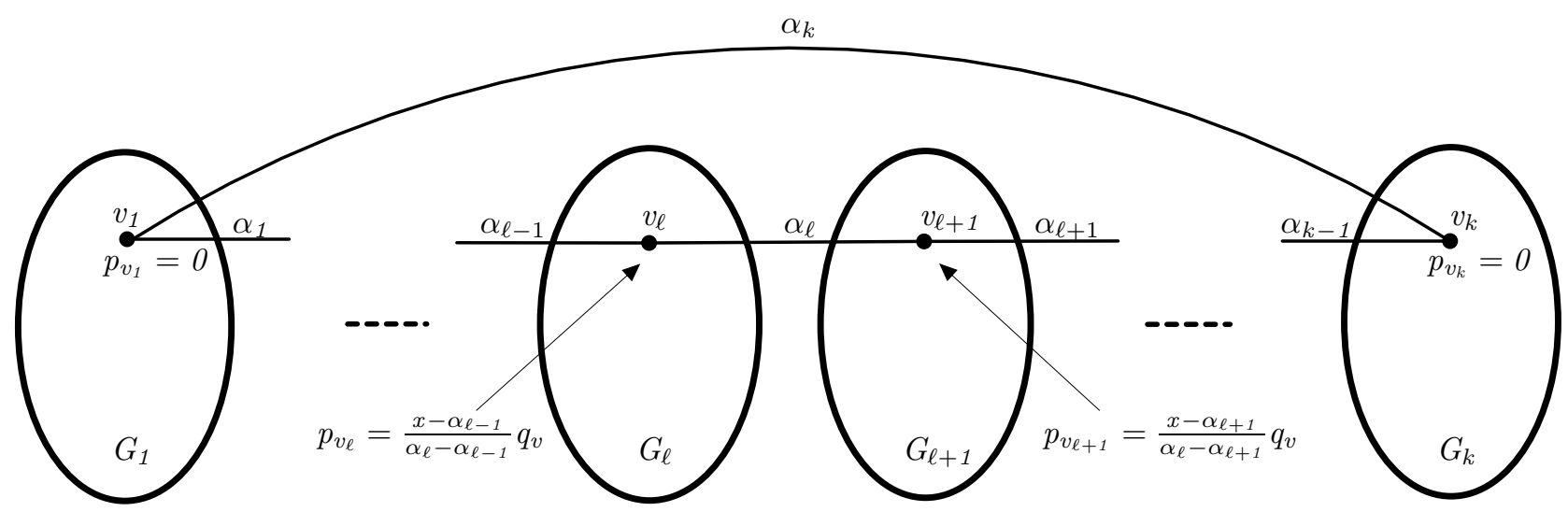

Figure 2: The vector $\left(q_{v}\right)_{v \in V(G)} \in B^{(r-1)}$ is used to construct $\mathbf{p}_{\mathbf{q}}^{(\ell)}=\left(p_{u}\right)_{u \in V\left(G \square C_{k}\right)}$ such that $\mathbf{p}_{\mathbf{q}}^{(\ell)} \in B_{\ell} \subseteq$ $W_{G \square C_{k}, c^{\prime}}^{r}$

Finally, consider a linear basis $B^{(r-2)}$ for $W_{G, c}^{r-2}$, and pick $\mathbf{q} \in B^{(r-2)}$ and let $\mathbf{q}=\left(q_{v}\right)_{v \in G}$. Note that $\operatorname{deg}\left(q_{v}\right) \leq \min \left\{r-2, \operatorname{deg}_{G}(v)\right\}-1$.

Define the vector $\mathbf{p}_{\mathbf{q}}^{(k)}=\left(p_{u}\right)_{u \in V\left(G \square C_{k}\right)}$ as $p_{v_{i}} \equiv 0$ for all $i \leq k-1$, and $p_{v_{k}}=\left(x-\alpha_{k-1}\right)\left(x-\alpha_{k}\right) q_{v}$. (See Figure 3). Note that $\operatorname{deg}\left(p_{v_{k}}\right) \leq 2+\operatorname{deg}\left(q_{v}\right)=\min \left\{r-2, \operatorname{deg}\left(v_{k}\right)-2\right\}+1=\min \left\{\operatorname{deg}\left(v_{k}\right), r\right\}-1$, and $p_{v_{k}}\left(\alpha_{k}\right)=p_{v_{k}}\left(\alpha_{k-1}\right)=0$. Hence the vector $\mathbf{p}_{\mathbf{q}}^{(k)}$ belongs to $W_{G \square C_{k}, c^{\prime}}^{r}$. Define $B_{k}=\left\{\mathbf{p}_{\mathbf{q}}^{(k)}: \mathbf{q} \in B^{(r-2)}\right\}$. Similar to above, the restriction of $\mathbf{p}_{\mathbf{q}}^{(k)}$ to $G_{k}$ shows that the vectors in $B_{k}$ are linearly independent.

We will show that the elements of $B_{1} \cup \ldots \cup B_{k}$ are linearly independent. We have already shown that the vectors in each individual $B_{i}$ are linearly independent. Next, note that if a vector $\left(p_{u}\right)_{u \in V\left(G \square C_{k}\right)} \in B_{j}$ for some $j \in[k]$, then $p_{v} \equiv 0$ for all $v \in \bigcup_{i=1}^{j-1} V\left(G_{i}\right)$, and $p_{v} \not \equiv 0$ for at least one vertex $v \in V\left(G_{j}\right)$. Thus $B_{j}$ does not intersect the span of $B_{j+1} \cup \ldots \cup B_{k}$. These show that $B_{1} \cup \ldots \cup B_{k}$ consists of exactly 


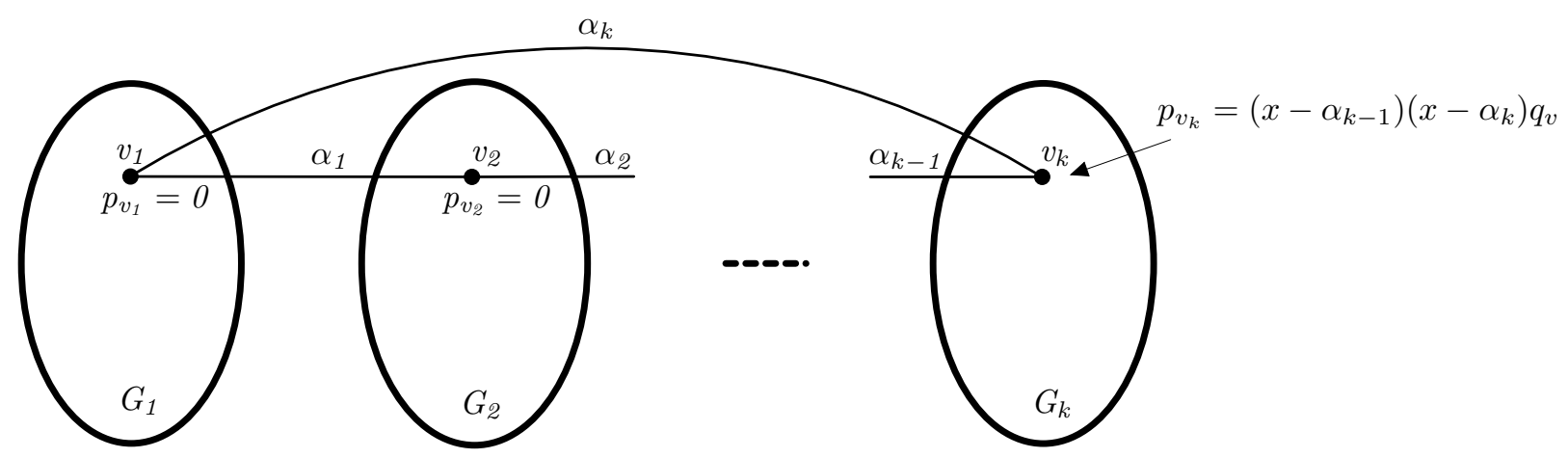

Figure 3: The vector $\left(q_{v}\right)_{v \in V(G)} \in B^{(r-2)}$ are used to construct $\mathbf{p}_{\mathbf{q}}^{(k)}=\left(p_{u}\right)_{u \in V\left(G \square C_{k}\right)}$ such that $\mathbf{p}_{\mathbf{q}}^{(k)} \in B_{k} \subseteq$ $W_{G \square C_{k}, c^{\prime}}^{r}$

$\operatorname{dim}\left(W_{G, c}^{r}\right)+(k-2) \operatorname{dim}\left(W_{G, c}^{r-1}\right)+\operatorname{dim}\left(W_{G, c}^{r-2}\right)$ linearly independent vectors.

To achieve the desired lower bound, we need to extend $B_{1} \cup \ldots \cup B_{k}$ to include an additional set of $d_{r-1}+k\left(d_{0}+\ldots+d_{r-2}\right)$ linearly independent vectors. First we show that no non-zero vector in $\operatorname{span}\left(B_{1} \cup\right.$ $\left.\ldots \cup B_{k}\right)$ evaluates to 0 on the colours of all the edges in $E\left(G_{1}\right) \cup \ldots \cup E\left(G_{k}\right)$. Indeed consider a non-zero $\mathbf{p}=\left(p_{u}\right)_{u \in V\left(G \square C_{k}\right)}$ in the span of $B_{1} \cup \ldots \cup B_{k}$, and consider the smallest $j$ such that there exists $u_{j} \in G_{j}$ with $p_{u_{j}} \not \equiv 0$. If $j=1$, then $p_{u_{j}}=q_{u}$ for some $\mathbf{q}=\left(q_{u}\right)_{u \in V(G)} \in \operatorname{span}\left(B^{(r)}\right)$, if $j \in[2, k-1]$, then $p_{u_{j}}=\frac{x-\alpha_{\ell-1}}{\alpha_{\ell}-\alpha_{\ell-1}} q_{u}$ for some $\mathbf{q} \in \operatorname{span}\left(B^{(r-1)}\right)$, and if $j=k$, then $p_{u_{j}}=\left(x-\alpha_{k-1}\right)\left(x-\alpha_{k}\right) q_{u}$ for some $\mathbf{q} \in \operatorname{span}\left(B^{(r-2)}\right)$. In the first case $\operatorname{deg}\left(q_{u}\right) \leq \min \{r, \operatorname{deg}(u)\}-1$, in the second case $\operatorname{deg}\left(q_{u}\right) \leq \min \{r-1, \operatorname{deg}(u)\}-1$, and in the third case $\operatorname{deg}\left(q_{u}\right) \leq \min \{r-2, \operatorname{deg}(u)\}-1$. Due to these degree restrictions, in neither of these cases $q_{u}$ can be a non-zero polynomial that evaluates to 0 on the colours of all the edges incident to it in $G$.

Hence to finish the proof, it suffices to find $d_{r-1}+k\left(d_{0}+\ldots+d_{r-2}\right)$ linearly independent vectors in $W_{G \square C_{k}, c^{\prime}}^{r}$ such that they all evaluate to 0 on the colours of $E\left(G_{1}\right) \cup \ldots \cup E\left(G_{k}\right)$. This together with the previous paragraph will guarantee that these vectors are independent from $B_{1} \cup \ldots \cup B_{k}$.

For every vertex $v$ in $G$ with $\operatorname{deg}_{G}(v)=r-1$, let $\mathbf{p}_{v}=\left(p_{u}\right)_{u \in V\left(G \square C_{k}\right)}$ be the vector defined as

$$
p_{v_{1}}(x)=\ldots=p_{v_{k}}(x):=\prod_{u: v u \in E(G)}\left(x-c_{v u}\right),
$$

and $p_{u_{1}}=\ldots=p_{u_{k}} \equiv 0$ for all $u \neq v$. Note, for $i \in[k], \operatorname{deg}\left(p_{v_{i}}\right)=r-1=\min \left\{r, \operatorname{deg}\left(v_{i}\right)\right\}-1$. So, it follows that $\mathbf{p}_{v} \in W_{G \square C_{k}, c^{\prime}}^{r}$.

Finally, for every vertex $v$ in $G$ with $\operatorname{deg}_{G}(v) \leq r-2$ and every $i_{0} \in[k]$, define the vector $\mathbf{p}_{v}^{i_{0}}=$ $\left(p_{u}\right)_{u \in V\left(G \square C_{k}\right)}$ as follows. Set $p_{u} \equiv 0$ for all $u \notin\left\{v_{i_{0}}, v_{i_{0}+1}\right\}$. Let $p_{v_{i_{0}}}$ be the unique polynomials of degree $\operatorname{deg}\left(v_{i_{0}}\right)-1=\operatorname{deg}_{G}(v)+1 \leq r-1$ that is equal to 1 on $c_{v_{i_{0}} v_{i_{0}+1}}^{\prime}$, and is equal to 0 on all the colours incident to $v_{i_{0}}$. Similarly, let $p_{v_{i_{0}+1}}$ be the unique polynomial of degree $\operatorname{deg}\left(v_{i_{0}+1}\right)-1=\operatorname{deg}_{G}(v)+1 \leq r-1$ that is equal to 1 on $c_{v_{i_{0} v_{i_{0}+1}}^{\prime}}$, and is equal to 0 on all the other colours incident to $v_{i_{0}+1}$. Obviously, $\mathbf{p}_{v}^{i_{0}}$ belongs to $W_{G \square C_{k}, c^{\prime}}^{r}$, and moreover the vectors

$$
\left\{\mathbf{p}_{v}: \operatorname{deg}_{G}(v)=r-1\right\} \cup\left\{\mathbf{p}_{v}^{i}: \operatorname{deg}_{G}(v)<r-1, i \in[k]\right\}
$$

are all linearly independent, and they evaluate to 0 on the colours of $E\left(G_{1}\right) \cup \ldots \cup E\left(G_{k}\right)$, and thus are independent from $B_{1} \cup \ldots \cup B_{k}$. Adding these $d_{r-1}+k\left(d_{0}+\ldots+d_{r-2}\right)$ vectors to the original $B_{1} \cup \ldots \cup B_{k}$ yields a linearly independent set of the desired size in $W_{G \square C_{k}, c^{\prime}}^{r}$.

Next we state the analogues of Proposition 4 and Theorem 5 for the Cartesian product of arbitrary graphs with paths. 
Proposition 6. Let $r>0, k \geq 2$ be integers, and $G=(V, E)$ be a graph. We have

$$
m_{e}\left(G \square P_{k}, r\right) \leq m_{e}(G, r)+(k-1) m_{e}(G, r-1)+d_{r-1}+(k-1)\left(d_{0}+\ldots+d_{r-2}\right),
$$

where $d_{t}$ denotes the number of vertices with degree exactly $t$ in $G$.

The proof of this proposition is almost identical to the proof of Proposition 4 . The only difference is that instead of an $(r-2)$-percolating set, we pick an $(r-1)$-percolating set for $G_{k}$.

Similar to Theorem 5 one can complement Proposition 6 with the following lower bound.

Theorem 7. Let $r>0, k \geq 2$ be integers, $G=(V, E)$ be a graph, and $c: E \rightarrow \mathbb{R}$ be a proper edge colouring of $G$. There exists a proper edge colouring $c^{\prime}$ of $G \square P_{k}$ for which

$$
\operatorname{dim}\left(W_{G \square P_{k}, c^{\prime}}^{r}\right) \geq \operatorname{dim}\left(W_{G, c}^{r}\right)+(k-1) \operatorname{dim}\left(W_{G, c}^{r-1}\right)+d_{r-1}+(k-1)\left(d_{0}+\ldots+d_{r-2}\right),
$$

where $d_{t}$ denotes the number of vertices with degree exactly $t$ in $G$.

The proof of this theorem is almost identical to the proof of Theorem 5 . The only difference is that $B_{k}$ is also constructed similar to $B_{2}, \ldots, B_{k-1}$.

\subsection{Exact bounds for Grids and Tori}

With Theorems 5 and 7 in hand, it is easy to prove a recursive formula for the sizes of the smallest percolating sets in tori and grids. We start with the tori.

Theorem 8. Let $d>0, a_{1}, \ldots, a_{d} \geq 3$, and $r \geq 0$ be integers. Denoting $G_{j}=\square_{i=1}^{j} C_{a_{i}}$ for $j \in[d]$, we have

$$
m_{e}\left(G_{d}, r\right)=m_{e}\left(G_{d-1}, r\right)+\left(a_{d}-2\right) m_{e}\left(G_{d-1}, r-1\right)+m_{e}\left(G_{d-1}, r-2\right)+\left\{\begin{array}{ll}
0 & r<2 d-1 \\
\prod_{i=1}^{d-1} a_{i} & r=2 d-1 \\
\prod_{i=1}^{d} a_{i} & r \geq 2 d
\end{array},\right.
$$

where $G_{0}$ is the graph with a single vertex.

Proof. Note that $G_{d-1}$ is a $2(d-1)$-regular graph, and thus Proposition 4 implies that $m_{e}\left(G_{d}, r\right)$ is bounded from above by the right hand side of (3). We will show that the other direction follows from Theorem 2 , Theorem 5 and a simple induction with the base case $m_{e}\left(G_{0}, r^{\prime}\right)=\operatorname{dim}\left(W_{G_{0}, c}^{r^{\prime}}\right)=0$ for every $r^{\prime}$. Indeed, assuming the existence of a coloring $c$ of $G_{d-1}$ with $m_{e}\left(G_{d-1}, r^{\prime}\right)=\operatorname{dim}\left(W_{G_{d-1}, c}^{r^{\prime}}\right)$ for every $r^{\prime}$, one can use Theorem 5 to obtain a proper edge colouring $c^{\prime}$ for $G_{d}$ with

$$
\operatorname{dim}\left(W_{G_{d}, c^{\prime}}^{r}\right) \geq m_{e}\left(G_{d-1}, r\right)+\left(a_{d}-2\right) m_{e}\left(G_{d-1}, r-1\right)+m_{e}\left(G_{d-1}, r-2\right)+\left\{\begin{array}{ll}
0 & r<2 d-1 \\
\prod_{i=1}^{d-1} a_{i} & r=2 d-1 \\
\prod_{i=1}^{d} a_{i} & r \geq 2 d
\end{array} .\right.
$$

This together with Theorem 2 completes the induction step and shows

$$
\operatorname{dim}\left(W_{G_{d}, c^{\prime}}^{r}\right)=m_{e}\left(G_{d}, r\right)=\text { R.H.S. of (3). }
$$

The case of the multidimensional grid can be proven similar to Theorem 8 , however, since the product of paths is not a regular graph, the formula is more complex.

Theorem 9. Let $d>0, a_{1}, \ldots, a_{d} \geq 2$, and $r \geq 0$ be integers. Denoting $G_{j}=\square_{i=1}^{j} P_{a_{i}}$, we have

$$
\begin{aligned}
m_{e}\left(G_{d}, r\right)= & m_{e}\left(G_{d-1}, r\right)+\left(a_{d}-1\right) m_{e}\left(G_{d-1}, r-1\right)+\sum_{S \subseteq[d-1],|S|=r-d} 2^{d-1-|S|} \prod_{i \in S}\left(a_{i}-2\right) \\
& +a_{d} \sum_{S \subseteq[d-1],|S|<r-d} 2^{d-1-|S|} \prod_{i \in S}\left(a_{i}-2\right) .
\end{aligned}
$$

where $G_{0}$ is the graph with a single vertex. 
The proof of this theorem proceeds similar to the proof of Theorem 8 . Here $\sum_{S \subseteq[d-1],|S|=r-d} 2^{d-1-|S|} \prod_{i \in S}\left(a_{i}-\right.$ 2 ) is the number of vertices of degree $r-1$ in $G_{d-1}$, and $\sum_{S \subseteq[d-1],|S|<r-d} 2^{d-1-|S|} \prod_{i \in S}\left(a_{i}-2\right)$ is the number of vertices of degree less than $r-1$. We leave this as an exercise to the interested reader.

\section{$3.2 \quad$ Hypercubes}

While Theorem 7 provides a recursive formula for the case of the grids, it is not clear whether there is a closed-form solution to this recursive formula. However for the special case of the hypercube, as it is shown in MN18, it is possible to obtain a closed-form formula. Let $d \geq r \geq 0$ be integers, and let $Q_{d}$ denote the $d$-dimensional hypercube. More formally the vertices of $Q_{d}$ are vectors $x \in\{0,1\}^{d}$ and two vertices are adjacent if they differ only in one coordinate.

Since $Q_{d}=P_{2}^{\square d}$, we have the following corollary to Theorem 9 that can be verified by a simple induction. This recovers the main result of MN18.

Corollary 10. We have

$$
m_{e}\left(Q_{d}, r\right)=\sum_{j=1}^{r}\left(\begin{array}{c}
d-j-1 \\
r-j
\end{array}\right) j 2^{j-1} .
$$

It is also possible to explicitly describe a set of percolating edges that achieves this bound. The recursive construction discussed in the previous section is as follows. Define $F_{r}\left(Q_{d}\right) \subseteq E\left(Q_{d}\right)$ in the following manner. If $r=0$, set $F_{r}\left(Q_{d}\right):=\emptyset$, and if $d=r$, set $F_{r}\left(Q_{d}\right):=E\left(Q_{d}\right)$. Otherwise let

$$
F_{r}\left(Q_{d}\right):=F_{r}\left(Q_{d-1}^{\prime}\right) \cup F_{r-1}\left(Q_{d-1}^{\prime \prime}\right),
$$

where $Q_{d-1}^{\prime}$ and $Q_{d-1}^{\prime \prime}$ are the two copies of $Q_{d-1}$ in $Q_{d}$ induced on the vertices $x=\left(x_{1}, \ldots, x_{d}\right)$ with $x_{d}=1$ and $x_{d}=0$, respectively. A simple induction proves the explicit description

$$
F_{r}\left(Q_{d}\right)=\left\{\left(x, \delta_{j} x\right): j \in[n], \sum_{i=1}^{j-1} x_{i} \geq d-r\right\},
$$

where $\delta_{j} x$ is the vector that is obtained from $x$ by flipping the value of the $j$-th coordinate. Note

$$
\left|F_{r}\left(Q_{d}\right)\right|=\sum_{j=1}^{r}\left(\begin{array}{c}
d-j-1 \\
r-j
\end{array}\right) j 2^{j-1}
$$

\section{Concluding remarks}

The polynomial method as it is used in Theorem 2 is applicable to a more general setting. Let $H=(V, E)$ be a hypergraph, and let $r$ be a nonnegative integer. Suppose that we initially infect a subset $F$ of the vertices. We start a process in which, at every step, if there is a hyperedge $S \in E$ that contains at least $r$ infected vertices, then the infection spreads to all the vertices in $S$. To prove a lower-bound for the size of the smallest percolating set for this percolation process we define a vector space similar to Definition 1

Definition 11. Let $r$ be a positive integer, $H=(V, E)$ be a hypergraph, and let $c: V \rightarrow \mathbb{R}$ be a vertex colouring of $H$ such that it assigns distinct colors to the vertices in each hyperedge. Let $W_{H, c}^{r}$ be the vector space of all vectors $\left(p_{e} \in \mathbb{R}[x]: e \in E\right)$, where $p_{e}$ 's are univariate polynomials such that

1. $\operatorname{deg}\left(p_{e}\right) \leq \min \{r,|e|\}-1$ for all $e \in E$;

2. $p_{e_{1}}\left(c_{v}\right)=p_{e_{2}}\left(c_{v}\right)$ for every pair $e_{1}, e_{2} \in E$ and for all $v \in e_{1} \cap e_{2}$. 
It is not difficult to see that, similar to Theorem 2, the dimension of the vector space $W_{H, c}^{r}$ is a lower bound for the size of the smallest percolating set in $H$.

This is more general than Theorem 2, as given a graph $G$, to recover Theorem 2, it suffices to consider the hypergraph $H$ with $V(H):=E(G)$, and hyperedges $S_{v}=\{u v: u v \in E(G)\}$ for $v \in V(G)$.

Another well-studied class of extremal percolation problems that falls into this framework is the special case of the $\mathcal{H}$-bootstrap process BBMR12, when $\mathcal{H}$ is a $k$-uniform hypergraph. In the $\mathcal{H}$-bootstrap process, we are given a hypergraph $\mathcal{H}$, and an initial set of infected vertices. At each time step, we infect a vertex $u$ if it lies in an edge of $\mathcal{H}$ in which all vertices other than $u$ are already infected. Note that if $\mathcal{H}$ is a $k$-uniform hypergraph, then this process is equivalent to the process described above with $r=k-1$. In particular, the graph bootstrap process of Bollobás Bol68 that was mentioned in the introduction is a special case of this. Recall that given graphs $G$ and $H$, and an initial set of infected edges, in the $H$-bootstrap process, at each time step, we infect an edge $e$ if it completes a new infected copy of $H$ in $G$. This is obviously an instance of the hypergraph $\mathcal{H}$-bootstrap process for the $|E(H)|$-uniform hypergraph $\mathcal{H}$ with $V(\mathcal{H}):=E(G)$, and hyperedges that correspond to the copies of $H$ in $G$.

\section{Acknowledgement}

We wish to thank Jonathan Noel for bringing our attention on the reference [LZ84 and the anonymous reviewer for pointing out the earlier application of polynomial method for bootstrap percolation in BBLN18]. The second author wishes to thank Noga Alon for valuable comments and discussions.

\section{References}

[BB06] József Balogh and Béla Bollobás. Bootstrap percolation on the hypercube. Probab. Theory Related Fields, 134(4):624-648, 2006.

[BBLN18] Paul N. Balister, Béla Bollobás, Jonathan Lee, and Bhargav P. Narayanan. Line percolation. Random Struct. Algorithms, 52:597-616, 2018.

[BBM10] József Balogh, Béla Bollobás, and Robert Morris. Bootstrap percolation in high dimensions. Combin. Probab. Comput., 19(5-6):643-692, 2010.

[BBMR12] József Balogh, Béla Bollobás, Robert Morris, and Oliver Riordan. Linear algebra and bootstrap percolation. J. Combin. Theory Ser. A, 119(6):1328-1335, 2012.

[Bol68] Béla Bollobás. Weakly $k$-saturated graphs. In Beiträge zur Graphentheorie (Kolloquium, Manebach, 1967), pages 25-31. Teubner, Leipzig, 1968.

[CLR79] J. Chalupa, P. L. Leath, and G. R. Reich. Bootstrap percolation on a bethe latice. Journal of Physics C: Solid State Physics, 12(1):L31-L37, 1979.

[GHM12] Janko Gravner, Alexander E. Holroyd, and Robert Morris. A sharper threshold for bootstrap percolation in two dimensions. Probab. Theory Related Fields, 153(1-2):1-23, 2012.

[LZ84] R. Lenormand and C. Zarcone. Growth of clusters during imbibition in a network of capillaries. In Kinetics of Aggregation and Gelation, page 177-180. Elsevier, Amsterdam, 1984.

[MN18] Natasha Morrison and Jonathan Noel. Extremal bounds for bootstrap percolation in the hypercube. Journal of Combinatorial Theory, Series A, 156:61-84, 052018. 